\title{
Parity Violation in Deep Inelastic Scattering in the JLab 12 GeV Era
}

\section{Paul E. Reimer*i}

Physics Division, Argonne National Laboratory, Argonne, Illinois 60439, USA

E-mail: reimereanl.gov

\begin{abstract}
Measurements of parity violation in electron scattering have yielded a wealth of information on the structure of the nucleon and on electroweak couplings. The upgrade of the CEBAF beam energy at Thomas Jefferson National Accelerator Facility (JLab) to a maximum of $12 \mathrm{GeV}$ will expand the range of kinematics in which parity violation measurements to include significantly more of the deep inelastic scattering (DIS) region, where a new information can be obtained on many interesting topics, including charge symmetry violation, higher twist contributions to proton structure and electroweak coupling constants. These coupling constants are related to $\sin ^{2} \theta_{W}$ in the framework of the Standard Model. To differentiate between these effects, measurements of parity violation in deep inelastic scattering (PVDIS) must be made over a large kinematic range in both $x_{\mathrm{Bj}}$ and $Q^{2}$. The SOLID collaboration has proposed the construction of a new, solenoidal spectrometer to make these measurements. This talk will describe these measurements and the SOLID spectrometer.
\end{abstract}

XVIII International Workshop on Deep-Inelastic Scattering and Related Subjects, DIS 2010 April 19-23, 2010

Firenze, Italy

\footnotetext{
*Speaker.

${ }^{\dagger}$ Representing the JLab SOLID, E05-007 and E12-07-102 Experiments
} 
The electroweak Standard Model has been remarkably successful in describing the unification of the electromagnetic and weak interaction at the level of a few parts per thousand. Even with this success, however, there are experimental hints [1] that the Standard Model is part of a larger framework. Precision, low-energy experiments are an important element in the effort to elucidate this larger framework [2]. The couplings between leptons and quarks may be sensitive to these extensions [3]. Within the Standard Model, these couplings vary with $\sin ^{2} \theta_{W}$. If their measured values deviate from Standard Model predictions, this will be a clear indication of processes not included in the Standard Model. The pattern of these deviations will help to clarify the larger framework governing the Standard Model.

Recent advances in the quality of polarized electron beams have made the measurement parity violating asymmetries in electron scattering an important probe of the weak force and the structure of the nucleon. Recently the HAPPEX [4] and G0 [5] experiments at Thomas Jefferson National Accelerator Facility (JLab) have used this parity violation to explore the effects of intrinsic strangeness in the proton. This talk will discuss a program of three measurements of parity violation in deep inelastic scattering (PVDIS) at JLab. These measurement include a $6 \mathrm{GeV}$ experiment in Hall A using standard equipment [6]; a $12 \mathrm{GeV}$ experiment in Hall $\mathrm{C}$ using baseline $12 \mathrm{GeV}$ equipment [7] and a $12 \mathrm{GeV}$ experiment requiring the construction of a large acceptance spectrometer [8].

The asymmetry in polarized electron scattering on unpolarized deuterium in DIS kinematics, $A_{\mathrm{DIS}}^{d}$, was first measured by Prescott et al. at SLAC [9] and served to the establish what is now known as the Standard Model of the electroweak interaction. This asymmetry can be expressed in terms of quark distribution functions of the target, and the couplings $C_{1 q}$ (axial electron $\times$ vector quark) and $C_{2 q}$ (vector electron $\times$ axial quark) which, within the context of the Standard Model, depend on $\sin ^{2} \theta_{W}[10]$ :

$$
\begin{array}{ll}
C_{1 u}=g_{A}^{e} g_{V}^{u}=-\frac{1}{2}+\frac{4}{3} \sin ^{2} \theta_{W}, & C_{1 d}=g_{A}^{e} g_{V}^{d}=\frac{1}{2}-\frac{2}{3} \sin ^{2} \theta_{W}, \\
C_{2 u}=g_{V}^{e} g_{A}^{u}=-\frac{1}{2}+2 \sin ^{2} \theta_{W}, & C_{2 d}=g_{V}^{e} g_{A}^{d}=\frac{1}{2}-2 \sin ^{2} \theta_{W} .
\end{array}
$$

Ignoring the intrinsic charmed quark contribution, on an isoscaler target such as deuterium, the PVDIS asymmetry, $A_{\mathrm{DIS}}^{d}$, expressed in terms of these couplings, is

$$
\begin{aligned}
A_{\mathrm{DIS}}^{d} & =-\left(\frac{3 G_{F} Q^{2}}{2 \sqrt{2} \pi \alpha}\right) \frac{\left(2 C_{1 u}-C_{1 d}\right)\left[1+R_{S}(x)\right]+Y\left(2 C_{2 u}-C_{2 d}\right) R_{v}(x)}{5+R_{v}(x)} \\
& \approx 10^{-4} Q^{2}\left(Q^{2} \text { in } \mathrm{GeV}^{2}\right)
\end{aligned}
$$

where the kinematical factor $Y$ is a function of the incident and scattered electron energy, $R_{v}(x)$ and $R_{S}(x)$ depend solely on the quark distributions.

In the near future, the $C_{1 q}$ coefficients will be well determined by the JLab QWeak [11] experiment combined with existing measurements of atomic parity violation in cesium [14]. The $C_{2 q}$ coefficients, however, are poorly known and measurement of parity non-conservation in DIS appears to be the best way to determine them. The current and projected knowledge of the $C_{1 q}$ and $C_{2 q}$ coupling constants is show in Fig. 1.

The interpretation of $A_{\mathrm{DIS}}^{d}$ in terms of the $C_{i q}$ couplings given by Eq. 2 is based on an assumption of quark scattering. With very high $Q^{2}$ and $W^{2}$, and moderate $x_{\mathrm{Bj}}$ this assumption should be valid. 

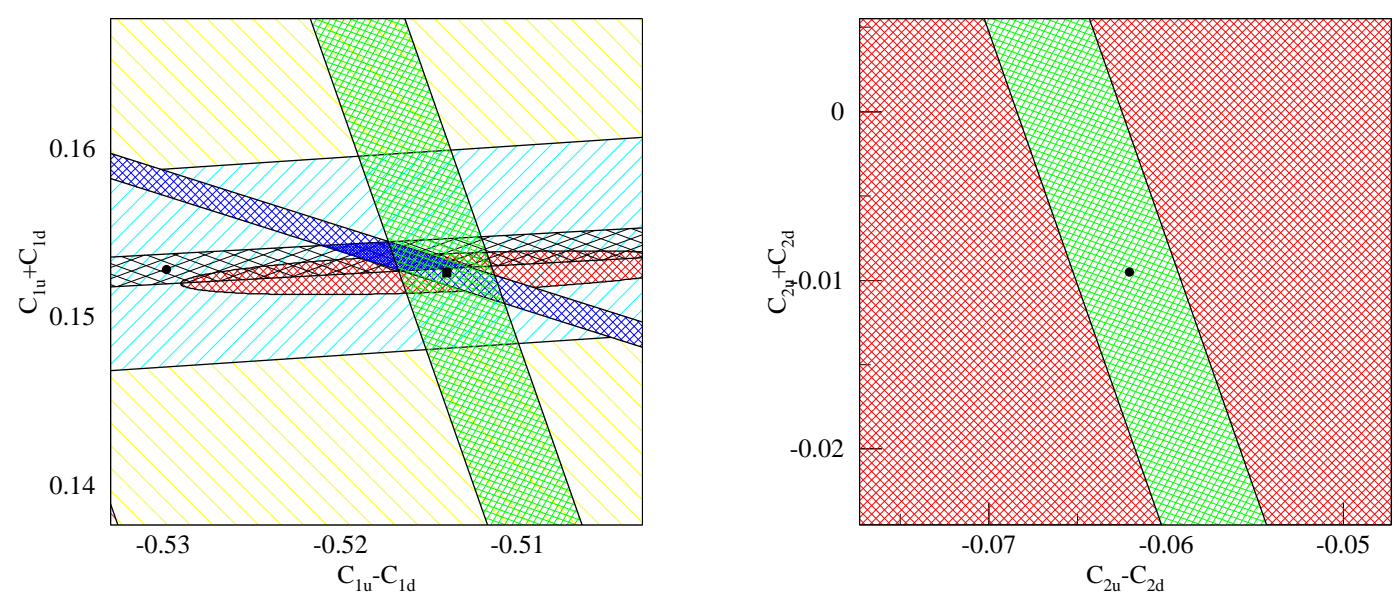

Figure 1: The current and future knowledge of the coupling constants $C_{1 q}$ (Left) $[14,11,9,12]$ and $C_{2 q}$ (Right) is shown. In both figures, the green diagonal band shows the expected uncertainty from the PVDIS program at JLab, and the red ellipse shows the results from the PDG's best fit [13]. Note that in the $C_{2}$ plane, the ellipse covers an area much larger than the entire graph which was plotted on the same scale as the $C_{1 q}$ couplings for comparison.

Table 1: This table shows the dependence of "new physics", CSV and Higher Twist on the kinematic variables $x_{\mathrm{Bj}}, Y$ (see Eq. 2) and $Q^{2}$.

\begin{tabular}{lccc}
\hline \hline & $x_{\mathrm{Bj}}$ & $Y$ & $Q^{2}$ \\
\hline New Physics & No & Yes & No \\
CSV & Yes & No & No \\
Higher Twist & Yes & No & Yes \\
\hline
\end{tabular}

Outside of these kinematics, additional hadronic effects may also contribute. These include charge symmetry violation (CSV) at the quark level and higher-twist effects. The dependence of $A_{\mathrm{DIS}}^{d}$ to these affects as a function of kinematic variables is shown in Tab. 1.

Charge symmetry violation in deuterium would manifest itself as an $x_{\mathrm{Bj}}$-dependent but $Q^{2}$ independent difference in $A_{\text {DIS }}^{d}$ from the predictions of Eq. 2. Recently, non-zero CSV effects have been allowed in some parton distribution fits. These fits tend to favor a small amount of CSV [15]. In addition, recent models of charge symmetry also tend to favor a similar, small CSV [16]. Based on these estimates size of the effect on $A_{\mathrm{DIS}}^{d}$ should be approximately $0.25 \%$ to $0.5 \%$.

The small amount of theoretical work which has been completed has shown that the effects of higher-twist on $A_{\mathrm{DIS}}^{d}$ are small, but possibly not negligible [17]. At the same time, there is currently no experimental information on the contribution of higher-twist to parity violating observables. An interesting aspect of higher-twist contributions in PVDIS is that only quark-quark correlations can will produce an observable effect in the first term in Eq. 2. PVDIS appears to be the only experimentally accessible to this important class of higher-twist terms. (For a summary of a recent workshop on higher-twist effects in PVDIS, please see Appdx. B in Ref. [8].) 
A series of three experiments at JLab using polarized electron beams from the CEBAF accelerator has been approved. These experiments include:

- the Hall A, $6 \mathrm{GeV}$ E05-007 [6], which collected data in late 2009 and will provide initial measurements of the $A_{\mathrm{DIS}}^{d}$;

- the Hall C $12 \mathrm{GeV}$ E12-07-102 [7], which will achieve better precision than the $6 \mathrm{GeV}$ measurement and reach deeper into the DIS region and

- the Hall A $12 \mathrm{GeV}$ SOLID [8], which will enable simultaneous measurement over a broad kinematic range in both $x_{\mathrm{Bj}}$ and $Q^{2}$.

The first of these experiments to run was the $6 \mathrm{GeV} \mathrm{JLab} \mathrm{Hall} \mathrm{A} \mathrm{experiment} \mathrm{which} \mathrm{collected}$ data in the fall of 2009 using the standard spectrometers and a specially developed "Counting DAQ”. The Counting DAQ relied on scalers with special logic to differentiate between pion and electron signals [18]. This experiment took data at two narrow kinematic points, $x_{\mathrm{Bj}}=0.25, Q^{2}=$ $1.11 \mathrm{GeV}^{2}$; and $x_{\mathrm{Bj}}=0.3, Q^{2}=1.90 \mathrm{GeV}^{2}$. These points were chosen to minimize the contribution of CSV and to be able to extract any $Q^{2}$ dependence of the asymmetry.

With the upgraded $12 \mathrm{GeV}$ CEBAF beam, a larger kinematic space becomes available within the DIS region. JLab has approved a second PVDIS experiment which use an $11 \mathrm{GeV}$ beam using baseline equipment. This measurement will take place in JLab Hall C and will be limited to a single kinematic point around $x_{\mathrm{Bj}}=0.34$ and $Q^{2}=3.3 \mathrm{GeV}^{2}$. The goal of this experiment is to achieve an overall uncertainty of $A_{\mathrm{DIS}}^{d}$ of $0.7 \%$.

To address the limited of acceptance that the baseline spectrometers will have, a new, solenoidal spectrometer has been proposed [8]. The design of this spectrometer is such that will will have sufficient kinematic acceptance to be able to cover $0.2<x_{\mathrm{Bj}}<0.75$ and $2<Q^{2}<10 \mathrm{GeV}^{2}$. The expected statistical sensitivity as a function of $x_{\mathrm{Bj}}$ and $Q^{2}$ is shown in Fig. 2. With this kinematic coverage, the differences between CSV, higher-twist and electroweak physics can be separated by fitting to the functional form [8]

$$
A_{\mathrm{DIS}}^{d}=A_{\mathrm{DIS}}^{\mathrm{EW}}\left[1+\beta_{\mathrm{HT}} \frac{1}{(1-x)^{3} Q^{2}}+\beta_{\mathrm{CSV}} x^{2}\right] .
$$

Because of this relatively large asymmetry, $A_{\mathrm{DIS}}^{d} \approx 300 \times 10^{-6}$ (depending on kinematics), many of the systematic effects, especially those related to beam induced false asymmetries, may be easily controlled to the required levels simply by using now standard techniques. The dominant systematic uncertainty in the measurement of $A_{\mathrm{DIS}}^{d}$ and the dominant technical challenge for this experiment is the measurement of the beam polarimetry. To address this, the SOLID collaboration has proposed using both a Compton polarimeter and an atomic hydrogen Møller polarimeter.

A program of three measurements of $A_{\text {DIS }}^{d}$ at JLab has been outlined. The interpretation of this measurement of $A_{\mathrm{DIS}}^{d}$ in terms of $C_{1 q}$ and $C_{2 q}$ depends on a theoretical and experimental understanding of the effects on higher-twist and charge symmetry violation on the measured asymmetry. While these effects are believed to be small, they have yet to be measured. Using the expected kinematic dependencies of these effects, they can be measured using a large acceptance spectrometer now proposed for JLab. Statistical precision of $0.5 \%$ can be quickly achieved for these measurements, because of the relatively large asymmetry. This work is supported in part by the U.S. Department of Energy, Office of Nuclear Physics, under Contract No. DE-AC02-06CH11357. 


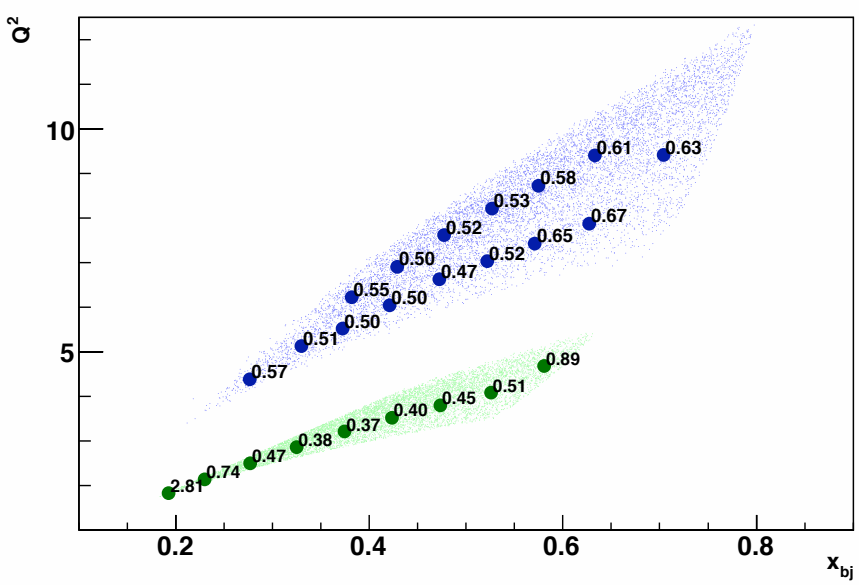

Figure 2: The expected statistical precision in $A_{\mathrm{DIS}}^{d}$ for bins in $x_{\mathrm{Bj}}$ and $Q^{2}$ are shown for two runs. The first run is for 120 "perfect" days at $11 \mathrm{GeV}$ and the second is for 60 days at $6.6 \mathrm{GeV}$, assuming a $50 \mu$ A beam and $85 \%$ polarization [8].

\section{References}

[1] M.S. Chanowitz, Phys. Rev. Lett. 87231802 (2001); M.S. Chanowitz, Phys. Rev. D66 073002 (2002).

[2] M.J. Ramsey-Musolf, Phys. Rept. 4561 (2008).

[3] P. Langacker, M. Lou and A.K. Mann, Rev. Mod. Phys. 6487 (1982).

[4] K.A Aniol et al. (The HAPPEX Collaboration) Phys. Rev. Lett. 96022003 (2006).

[5] D.S. Armstrong et al. (G0 Collaboration) Phys. Rev. Lett. 95092001 (2005).

[6] X. Zheng et al. (JLab E05-007 PVDIS Collaboration)Proposal to JLab PAC (2004).

[7] P. Reimer et al. (JLab E12-07-102 PVDIS Collaboration)Proposal to JLab PAC (2006).

[8] P. Souder et al. (The JLab SOLID Collaboration) Proposal to JLab PAC (2010); P. Souder, in DIS2008 7-11 Apr 2008, London, England, http://dx.doi.org/10.3360/dis.2008.243.

[9] C.Y. Prescott et al. Phys. Lett. B77 347 (1978); C.Y. Prescott et al. Phys. Lett. $B 84524$ (1979).

[10] R.N. Cahn, F.J. Gilman, Phys. Rev. D17 1313 (1978); J.D. Bjorken, Phys. Rev. D18 3239 (1978).

[11] R. Carlini et al (The QWeak Collaboration) Proposal to JLab PAC (2001).

[12] P.A. Vetter et al. Phys. Rev. Lett. 742658 (1995); A.I. Milstein, O.P. Sushkov, and I.S. Terekhov, Phys. Rev. Lett. 89283003 (2002).

[13] C. Amsler et al. (Particle Data Group), Phys. Lett. B667 1 (2008).

[14] S.C. Bennett, C.E. Wienman, Phys. Rev. Lett. 822484 (1999).

[15] A.D. Martin et al Eur. Phys. J. C35 325 (2004).

[16] E.N. Rodionov, A.W. Thomas and J.T. Londergan, Mod. Phys. Lett. A9 1799(1994).

[17] P. Castorina and P.J. Mulders, Phys. Rev. D31 2760 (1985); S. Fajfer adn R.J. Oakes, Phys. Rev. D30 1585 (1984).

[18] R. Subdei et al, Nucl. Instr. Meth. in preparation. 